\section{GREQAM}

Groupement de Recherche en Economie Quantitative d'Aix-Marseille - UMR-CNRS 6579 Ecole des Hautes Etudes en Sciences Sociales Universités d'Aix-Marseille II et III
Document de Travail n'2010-25

July, 2010

THRESHOLD VALUE: HOW BINDING THRESHOLDS AFFECT THE OUTCOME OF A NEGOTIATION

\author{
Sylvie THORON
}




\title{
Threshold Value: how binding thresholds affect the outcome of a negotiation
}

\author{
Sylvie Thoron*
}

February 23, 2010

\begin{abstract}
The objective of this paper is to give the prospective outcome or "prospect" of a negotiation when the players' value can only be realized if a certain threshold is reached. Thresholds can be of different forms such as a certain number of players, a certain coalition of players or a certain total level of contribution. A value is proposed, which is defined for a given game and a given threshold. It is derived from a decomposition of the Shapley value.

Journal of Economic Literature classification numbers: C71, C78.
\end{abstract}

Keywords: threshold, commitment, Shapley value, coalition structures, weights.

*GREQAM and University of Toulon. 


\section{Introduction}

Think of a group of partners who wish to invest in a project. They meet around a table to discuss the total amount of money they need to realize this project and the individual contribution that each of them should make. However, whatever the result of this discussion, the different participants are not able to put their contributions on the table at the end of the meeting. Each one goes back home and the day after starts to raise the money required through a credit or disaving. However, for some or all of the partners the project is only attractive if certain conditions are fulfilled: a minimum amount of money must be raised, certain key-partners must participate. Therefore, the group has to negotiate a conditional outcome, a sharing of the amount of money needed, which will only be implemented if the conditions are fulfilled. The partners may be satisfied by this conditional outcome: they will be willing to participate if the conditions are fulfilled and they will not be obliged to participate if the conditions are not met. However, it may be that other partners, who are more involved in the project, would like to ensure that these conditions will be fulfilled. In this situation, the result of the negotiation will certainly depend on the partners' different levels of involvement.

What sort of theoretical tools can we use to analyse this sort of procedure? Clearly, the problem is to analyse the ex-ante effect of a threshold on the result of a negotiation. There is a large literature on thresholds using a game theoretical and experimental approach. Thresholds are often invoked in the literature on games of contribution to a public good, for example. It is argued there that they are incentive compatible (cf. Bagnoli and Lipman (1989), Palfrey and Rosenthal (1984)).

A first problem concerns the definition of thresholds. In the voluntary contribution to a public good literature, the threshold is defined as a minimum level of contributions. However, in many real economic situations the contribution to a public good and the threshold are not defined in monetary terms. Consider the example of international environmental agreements. After the agreement has been defined and signed, the different signatories have to ratify the treaty. The ratification phase is then governed by a minimum participation constraint which plays the role of a threshold. Depending on the treaty in question, this threshold is defined in very different ways: as a set of countries, a number of countries or a percentage of the targeted polluting substances (cf Barret (2003) pages $165-195)$.

Another problem is that, in this literature, the focus is on the problem of efficiency in a non-cooperative framework. When a certain "level" of contributions is needed to provide a public good, at the equilibrium of the game of private contribution, the "sum" of contributions is efficient. However, can we say something about the distribution of these contributions amoung the participants and can they be asymmetric?

It is often useful to adopt a cooperative approach to deal with asymmetries. 
Cooperative game theory proposes alternative tools, solution concepts, to share a value or a cost based on different principles. For example, the Shapley value is based on the principle that each player is rewarded according to her incremental worth, that is, to her contribution to the worth of a given coalition. Then, for a given player, her Shapley value is her expected incremental contribution, when it is assumed that the different orders in which the partners join the coalition are equally probable. It has been argued that the Shapley value allows one "to evaluate the players' prospects"- Hart and Kurz (1983) p1047. Let me also quote Shapley (1953 p. 307):

"At the foundation of the theory of games is the assumption that the players of a game can evaluate, in their utility scales, every "prospect" that might arise as a result of a play. In attempting to apply the theory to any field, one would normally expect to be permitted to include, in the class of "prospects," the prospect of having to play a game. The possibility of evaluating games is therefore of critical importance."

The idea that a value can be interpreted as the expected outcome of a negotiation has been also explicitly developed by Hart and Kurz (1983). However, they kept a purely cooperative and axiomatic approach in their definition of the CS-value (coalition structure value) that they propose. Later on, the non cooperative literature has proposed different ways of proving what was originaly just an interpretation (see for example Perez-Castrillo and Wettstein (2001) or Maskin (2004)). Indeed empirical studies had already corroborated this interpretation (see for example Littlechild and Thompson (1979)). In this paper, we will not try to propose a non-cooperative game to describe the negotiation. We will adopt the cooperative approach but we will keep in mind the interpretation that the value corresponds to an evaluation of players' prospects.

Another important point about this interpretation of the Shapley value, concerns the weights of the different players in the negotiation. The value is defined for a given game $v$ which represents the contribution of each coalition and $v(N)$, with $N$ a arrier of the game, is then the amount the players have to share. However, $v$ cannot represent all the elements which could play a role in the negotiation. These elements are incorporated in the weights that the different players have in the negotiation. Kalai and Samet (1988) say that: "The weights should be determined by considering such factors as bargaining ability, patience rates, or past experiences". In the Shapley value, everything is considered to be incorporated in the game $v$ and these weights are symmetric. However, Shapley (1953) also introduced the concept of a family of Shapley values which has generated an extensive literature. Kalai and Samet (1988) have proposed an axiomatic characterization of the family of Shapley values. A weighted Shapley value is defined for a given, ordered, coalition structure and a vector of exogeneous weights. Yet, the underlying common feature of the different weighted Shapley values which satisfy Kalai and Samet's Axioms does not necessarily exhaust all the possible ways in which weights can be used. 
Indeed, Hart and Kurz (1983) have proposed a value which does not satisfy these Axioms. However, Hart and Kurz's coalition structure value intuitively represents the Shapley value players can expect when they form coalitions:

"Our view is that the reason coalitions form is not in order to get their worth, but to be in a better position when bargaining with the others on how to divide the maximal amount available." (p. 1052)

The coalition structure modifies the different players' weights in the negotiation and the resultant sharing of $v(N)$ in comparison with the Shapley value. But contrary to what is required for a weighted Shapley value, here the coalition structure is not ordered. As in the case of the weighted Shapley value, the coalition structure modifies the probability of the different orders defined on the set of players. But in their definition of the Coalition Structure value, Hart and Kurz (1983) do not need to order the different coalitions. They assume that, the only orders to appear are orders consistent with the coalition structure: the orders in which the coalitions appear as blocks.

In this paper, I propose a value for a given threshold. A threshold can be represented by a coalition but in a general framework could be a set of coalitions which can reach the threshold. The problem I want to address concerns the delicate situations in which players do not know, at the moment at which they have to negotiate, which coalition will actually form. They only know, through the threshold rule, which coalitions may form.

The threshold value (T-value) I am going to propose, which is defined for any game and for any given threshold is derived from a decomposition of the Shapley value. By comparing the T-value with the Shapley value, I show how the threshold can distort the sharing rule in favour of some players and at the expense of others.

The paper is organized as follows. Section 2 introduces a decomposition of the Shapley value which is used in Section 3 where the threshold value is presented for a specific definition of thresholds. It comes with a heuristic description and an axiomatization. I will also discuss in this section, the relationship between this value, the family of weighted Shapley value (Kalai and Samet (1988)) and the CS-value proposed by Hart and Kurz (1983). The properties of the value are then analysed in the last section when the definition of thresholds is generalized. I give examples of what I call neutral and non neutral thresholds.

\section{A decomposition of the Shapley value}

In this section, both well known and new concepts and results will be presented and these will be used in the following section. Let $U$ denote the universe of players. A coalition of players is a subset $S \subset U$. A game $v$ is a function which associates a real number $v(S)$ to each coalition $S \subset U, v(S)$ represents the 
total payoff or worth the coalition $S$ can get in game $v$. I will only consider games which are superadditive, that is, for two disjoint coalitions $S$ and $T \subset U$, $v(S)+v(T) \leq v(S \cup T)$. The incremental worth of player $i$ to coalition $S, i \notin S$, is $v(S \cup\{i\})-v(S)$. Players $i, j$ are said to be symmetric with respect to game $v$ if they make the same incremental worth to any coalition, i.e., for each $S \subset U$ with $i, j \notin S, v(S \cup\{i\})=v(S \cup\{j\})$. A finite carrier of $v$ is any set $N \subseteq U$ with $v(S)=v(N \cap S)$ for all $S \subseteq U$. A dummy player in game $v$ is a player $i$ whose incremental worth to each coalition is zero, i.e., $v(S \cup\{i\})-v(S)=0$ for every $S \subset U$. Therefore, if $N$ is a finite carrier of $v$, all players in $U \backslash N$ are dummies. A value is an operator $\varphi$ that assigns to each game $v$ and each player $i$ in $U$ a payoff $\varphi_{i}(v)$. The Shapley value is characterized by three very simple axioms.

Axiom 1 Efficiency. For each finite carrier $N$ of $v$,

$$
\sum_{i \in N} \varphi_{i}(v)=v(N)
$$

Axiom 2 Additivity. For any two games $v$ and $u, \varphi_{i}(v+u)=\varphi_{i}(v)+\varphi_{i}(u)$

Axiom 3 Symmetry. If players $i, j$ are symmetric with respect to game $v$, then

$$
\varphi_{i}(v)=\varphi_{j}(v) .
$$

The Shapley value for player $i$ in game $v$ can be calculated as her expected incremental value over all possible orders defined on any given carrier $N$ of $v$, under the assumption that each order appears with the same probability. Denote by $R(N)$ the set of all possible orders defined on $N$. In a given order $r \in R(N)$ each player $i \in N$ is associated with a ranking $r_{i}$. Consider the coalition of all the players who have a ranking at most as high as $i$ in this specific order: $S=\left\{j: r_{j} \leq r_{i}\right\}$. Then, in order $r$, player $i$ 's incremental worth is $v(S)-v(S \backslash i)$. Note that, for notational convenience I will sometimes write singleton $\{i\}$ just as $i$. If all the orders in $R(N)$ are considered to be equiprobable the expected incremental value of player $i$ is her Shapley value:

$$
\varphi_{i}(v)=\frac{\sum_{S \subset N, i \in S}(s-1) !(n-s) !(v(S)-v(S \backslash i))}{n !}
$$

Now, for each coalition $M \subset N$, which is not a carrier of $v$, we can define a $M$-game $v^{M}$ for which $M$ is a carrier:

$$
\begin{aligned}
& v^{M}(S)=v(S), S \subset M \\
& v^{M}(S)=v(S \cap M) \text { otherwise }
\end{aligned}
$$

Then, given $r(M)$ the set of all possible orders on $M$, the Shapley value of $v^{M}$ is for each $i$ in $M$ :

$$
\begin{aligned}
\varphi_{i}\left(v^{M}\right) & =\frac{\sum_{S \subset N, i \in S}(s-1) !(m-s) !\left(v^{M}(S)-v^{M}(S \backslash i)\right)}{m !} \\
& =\frac{\sum_{S \subset M, i \in S}(s-1) !(m-s) !(v(S)-v(S \backslash i))}{m !}
\end{aligned}
$$


This can be interpreted as the value obtained by the members of coalition $M$, when they share $v(M)$ without taking into account the players who are not in $M$. Now, for each game $v^{M}$, we define what we call an incremental game $v^{M *}$ as follows. For each given coalition $M \subset U, v^{M *}$ gives the incremental worth of each subset $S$ of $M$ to $N \backslash M$, where $N$ is any carrier of $v$ :

$$
\forall S \subset M, v^{M *}(S)=v((N \backslash M) \cup S)-v(N \backslash M)
$$

Note that, if $M$ is a carrier of game $v, N \backslash M$ is a coalition of dummies and $v^{M *}(S)=v(S)$. In general however, since the game is superadditive, $v^{M *}(S) \geq$ $v(S)$. The Shapley value for game $v^{M *}$ and each player $i$ in $M$ is, for each carrier $N$ of $v$ :

$$
\begin{aligned}
\varphi_{i}\left(v^{M *}\right) & =\frac{\sum_{S \subset M, i \in S}(s-1) !(m-s) !\left(v^{M *}(S)-v^{M *}(S \backslash\{i\})\right)}{m !} \\
& =\frac{\sum_{S \subset M, i \in S}(s-1) !(m-s) !(v(S \cup(N \backslash M))-v(S \backslash\{i\} \cup(N \backslash M)))}{m !}
\end{aligned}
$$

This can be interpreted as the value obtained by the members of coalition $M$, when they share $v(N)-v(N \backslash M)$, which can be called, by extension, the incremental worth of coalition $M$. In other words, this is the value which is calculated for each $M$-member, assuming that a coalition $N \backslash M$ got its worth $v(N \backslash M)$ already and taking into account all possible orders of players in $M$ when they join the coalition $N \backslash M$. Of course, if $M$ is a carrier of $v, \varphi_{i}\left(v^{M *}\right) \equiv \varphi_{i}(v)$, the Shapley value of player $i$ in the game $v$.

Example 1 An obvious case is that in which $M$ is a singleton $\{i\}$. Then, $\varphi_{i}\left(v^{\{i\}}\right)=v(\{i\})$ and $\varphi_{i}\left(v^{\{i\} *}\right)=v(N)-v(N \backslash\{i\})$ is player $i$ 's incremental worth to $N$, a carrier of the game.

Example 2 Let $N=\{1,2,3,4\}$. Then,

$$
\begin{aligned}
\varphi_{1}\left(v^{\{1,2\}}\right) & =\frac{v(\{1\})+v(\{1,2\})-v(\{2\})}{2 !} \\
\text { and } \varphi_{3}\left(v^{\{3,4\} *}\right) & =\frac{v(\{1,2,3\})-v(\{1,2\})+v(N)-v(N \backslash\{3\})}{2 !}
\end{aligned}
$$

Example 3 Let $N=\{1,2,3\}$ and $\varphi_{i}\left(v^{\{i\}}\right)=v(\{i\})$,

$$
\varphi_{i}\left(v^{\{i, j\} *}\right)=\frac{v(N)-v(N \backslash\{i\})+v(\{i, k\})-v(\{k\})}{2 !} .
$$

Note that, we can write in this case:

$$
\begin{gathered}
\varphi_{i}\left(v^{\{i\}}\right)+\varphi_{i}\left(v^{\{i, j\} *}\right)+\varphi_{i}\left(v^{\{i, k\} *}\right)= \\
\frac{2 v(\{i\})+2(v(N)-v(N \backslash\{i\}))+v(\{i, k\})-v(\{k\})+v(\{i, j\})-v(\{j\})}{2 !} \\
=3 \varphi_{i}(v)
\end{gathered}
$$


Now, if we consider $\varphi_{i}\left(v^{\{i\} *}\right)=v(N)-v(N \backslash\{i\})$ and $\varphi_{i}\left(v^{\{i j\}}\right)=\frac{v(i j)-v(j)+v(\{i\})}{2 !}$, we can also write:

$$
\begin{gathered}
\varphi_{i}\left(v^{\{i\} *}\right)+\varphi_{i}\left(v^{\{i, j\}}\right)+\varphi_{i}\left(v^{\{i, k\}}\right) \\
=\frac{2 v(\{i\})+2(v(N)-v(N \backslash\{i\}))+v(\{i, k\})-v(\{k\})+v(\{i, j\})-v(\{j\})}{2 !} \\
=3 \varphi_{i}(v)
\end{gathered}
$$

The following Proposition shows that indeed, there is a general relationship between the Shapley value for a game $v$, characterized for any carrier $N$ of $v$ and the Shapley values for games $v^{M}$ and $v^{M *}$ in which $M$ is not a carrier of $v$. For each carrier $N$ of $v$, we denote by $C_{m}$ the class of coalitions $M \subset N$, of the same size $m$ :

$$
C_{m}=\{M \subset N: \# M=m\}
$$

We can prove the following relationship:

Proposition 1 For each carrier $N$ of $v$ and each given class $C_{m}, 1 \leq m \leq n$ :

$$
\varphi_{i}(v)=\frac{m !(n-m) !}{n !}\left[\sum_{\substack{M \in C_{m} \\ i \in M}} \varphi_{i}\left(v^{M}\right)+\sum_{\substack{M \in C_{m} \\ i \notin M}} \varphi_{i}\left(v^{N \backslash M *}\right)\right]
$$

Proof. cf Appendix.

\section{The $T$-value for a given coalition}

In this Section, I propose a value for a given game $v$ and a given threshold. I will consider that a threshold is simply a coalition $T \subset U$, which is not a carrier of $v$ but satisfies a specific condition: the sharing of $v(N)$ with $N$ any carrier of $v$, can only be implemented if the $T$-members are involved. A $T$-value of a game $v$ with a threshold $T$ is then an operator that assigns to each player $i$ in $U$ a payoff $\phi_{i}(v, T)$. The $T$-value, like the Shapley value, is efficient. The players efficiently share $v(N)$. But now, in each order, $v(N)$ is split between the worth realized by the first coalition to reach the threshold, let us say coalition $S$ which is such that $T \subset S$, and the incremental worth of the remaining coalition $N \backslash S$. In the following sub-section, I give a heuristic description of a mechanism which would produce the $T$-value of a game $v$ as the expected outcome.

\subsection{Heuristic approach}

Following the usual heuristic description of the Shapley value, players have to meet in a bargaining room to share the worth of the grand coalition. They arrive sequentially and the order in which they do so is determined by chance, with all arrival orders equally probable. Each player, when she enters the room, 
demands and is promised the amount which her participation contributes to the worth of the grand coalition.

Here, I modify this description by introducing two rooms: the waiting room and the bargaining room. The participants arrive sequentially and in a random order in the waiting room. But there is nothing to share before the coalition formed by the players in the waiting room has reached the threshold. When the last player necessary to reach this threshold enters the waiting room, the door is closed from outside and the usual process is followed by the present players. Players arrive sequentially and in a random order in the bargaining room. Each player from the waiting room, when she enters the bargaining room, demands and is promised the amount which her participation contributes to the worth of the coalition. For each coalition $S$ which reaches the threshold, the value $\varphi_{i}\left(v^{S}\right)$ represents what player $i \in S$ can obtain in this negotiation.

When the waiting room is empty again, its outside door is reopened and the remaining players arrive sequentially and go straight to the bargaining room in order to demand the amount which their adherence contributes to the worth of the grand coalition. For each coalition $S$ which reaches the threshold and to which player $i$ does not belong, the value $\varphi_{i}\left(v^{N \backslash S *}\right)$ represents what player $i$ can obtain in this negotiation.

\subsection{Definitions}

For each carrier $N$ of $v$, consider the set $\Theta^{T}=\{S \subset N: T \subset S\}$, this is the set of coalitions which satisfy threshold $T$. We will say that player $i \in N$ is necessary for the threshold to be reached if $i \in T$. A symmetric game $v_{K}$, is defined, for a given coalition $K \subset U$, as follows:

$$
\begin{aligned}
v_{K}(S) & =1 \text { if } K \subset S \\
& =0 \text { otherwise }
\end{aligned}
$$

In what follows, I give a probabilistic interpretation of thresholds. Remember that $r(N)$ is an order defined on the set of players and for a given order $r(N)$, each player $i$ is associated with a ranking $r_{i}$. For a given threshold $T \subset N$ and a given order $r(N)$, consider the coalitions $S \subset N$, which satisfy the three following conditions:

(i) $\quad S \in \Theta^{T}$

(ii) All the players who belong to coalition $S$ arrive successively in $r(N)$ : given $i \in S$, such that $r_{i}=\operatorname{Min}_{k \in S} r_{k}$ and $j \in S$ such that $r_{j}=\operatorname{Max} x_{k \in S} r_{k}$, $\forall k \in S, r_{j} \geq r_{k} \geq r_{i}$. In other words, think about $S$ as a block in the order $r(N)$.

(iii) One of the $S$-members arrives first in the order. In other words $r_{i}=\operatorname{Min}_{k \in S} r_{k}=1$.

For a given threshold $T \subset N$, to each order $r(N)$ corresponds a unique coalition $S$, which satisfies the three previous conditions and has the smallest number of members. But each coalition $S$ may correspond to several orders. This defines an injective but not surjective application from the set of orders 
$r(S)$ to the set of coalitions $\Theta$. Denote by $\alpha_{S}$ the number of orders associated with coalition $S, \alpha_{S}=(n-s) !(s-1) ! t$ : this is the number of orders in which the $S$-members all arrive before the others, but among them the last to arrive is a $T$-member. In other words, in the order $r(N)$, the threshold is reached when the last $T$-member has arrived and all the players who have a smaller ranking constitute coalition $S$, associated with this order. Note that as a consequence of the injective application, we have: $\sum_{S \in \Theta^{T}} \alpha_{S}=n$ !

\subsection{Axiomatization}

I will now argue that the following function can be considered as an expected Shapley value:

Consider $N$, any finite carrier of $v$, and a threshold $T \subset N$,

$$
\begin{aligned}
& \left.\phi_{i}(v, T)=\frac{\sum_{S \in \Theta^{T}, i \in S} \alpha_{S} \varphi_{i}\left(v^{S}\right)+\sum_{S \in \Theta^{T}, i \notin S} \alpha_{S} \varphi_{i}\left(v^{N \backslash S *}\right)}{n !}, \forall i \in N \backslash T 1\right) \\
& \phi_{i}(v, T)=\frac{\sum_{S \in \Theta^{T}, i \in S} \alpha_{S} \varphi_{i}\left(v^{S}\right)}{n !}, \forall i \in T
\end{aligned}
$$

where $\alpha_{S}=(n-s) !(s-1) ! t$, and $\varphi_{i}\left(v^{S}\right)$ and $\varphi_{i}\left(v^{N \backslash S *}\right)$ are defined in the previous section. This function is obtained using the probabilistic description of thresholds presented in sub-section 3.2. Consider a given threshold $T$. For each order $r(N)$, the associated coalition $S$ is the set of players who first reach the threshold $T$ and the Shapley value is applied to this set of players. Then, in order to share $v(N)-v(S)$ among the $N \backslash S$ other players, the Shapley value is applied to the incremental game $v^{N \backslash S *}$. The previous function is therefore an expected Shapley value. Note that when $T$ is a carrier of $v$, we can write $\Theta^{T}=\{T\}$ and $\alpha_{T}=(t-1) ! t=t$ !. Therefore, when $T$ is a carrier of $v, \phi_{i}(v, T)=\varphi_{i}(v)$. For notational convenience I may write, for a given coalition $M, \phi_{M}(v, T)$ for $\sum_{i \in M} \phi_{i}(v, T)$ and $\varphi_{M}(v, T)$ for $\sum_{i \in M} \varphi_{i}(v, T)$.

Consider the following Axioms defined for any function $\phi(v, T)$, with $T \subset$ $N, N$ any carrier of $v$.

Axiom 4 Efficiency*. For each finite carrier $N$ of $v$,

$$
\sum_{i \in N} \phi_{i}(v, T)=v(N)
$$

Axiom 5 Additivity*. For any two games $v$ and $u$ :

$$
\phi_{i}(v+u, T)=\phi_{i}(v, T)+\phi_{i}(u, T)
$$

Axiom 6 Symmetry*. If players $i, j$ are symmetric with respect to $v$ and $i, j \in$ $T$ or $i, j \notin T$, then

$$
\phi_{i}(v, T)=\phi_{j}(v, T)
$$


Axiom 7 Commitment cost. For any symmetric game $v_{K}$,

$$
\phi_{T}\left(v_{K}, T\right)=\frac{t}{k} \phi_{T}\left(v_{K}, K\right)
$$

Note that $\phi_{i}\left(v_{K}, K\right)$ is the function defined for a threshold $K$ that is a carrier of a symmetric game. In that case, as noted above $\phi_{i}\left(v_{K}, K\right)=\varphi_{i}(v)=\frac{1}{k}$. Taking this case as a benchmark, the Commitment cost Axiom shows how the threshold modifies the value in a symmetric game. In a symmetric game $v_{K}$, all the players in $K$ are necessary to realize a worth. In that case, when there is no threshold, the value gives to each of the $k$ players an equal share of $v(K)$. Then, when the threshold is introduced, the Axiom says that the players who are necessary for the threshold to be reached only receive a proportion $\frac{t}{k}$ of what they have without the threshold. An intuitive interpretation of this Axiom is that the players who are necessary for the threshold to be reached are committed to the sharing, while the others are not. All the players in $K$ will participate in negotiating the sharing of $v(K)$, but the players who are necessary for the threshold to be reached have a leadership commitment. They will initiate the sharing process and the cost of this is that they will be restricted to sharing the worth of the coalition which has been necessary to reach the threshold $v(N \backslash M)$, whilst the remaining players can share the incremental worth $v(N)-v(M)$.

Theorem 1 There is a unique value which satisfies Efficiency*, Additivity*, Symmetry* and Commitment cost and it is defined by Equalities (1) and (2).

Proof. First step: I prove that the function defined by Equalities (1) and (2)satisfies the four Axioms.

(i) It is efficient. Sum the values of the function over a finite carrier $N$ :

$$
\begin{aligned}
\sum_{i \in N} \phi_{i}(v, T) & =\sum_{i \in N} \frac{\sum_{S \in \Theta^{T}, i \in S} \alpha_{S} \varphi_{i}\left(v^{S}\right)+\sum_{S \in \Theta^{T}, i \notin S} \alpha_{S} \varphi_{i}\left(v^{N \backslash S *}\right)}{n !} \\
& =\frac{\sum_{S \in \Theta^{T}}\left[\sum_{i \in S} \alpha_{S} \varphi_{i}\left(v^{S}\right)+\sum_{i \notin S} \alpha_{S} \varphi_{i}\left(v^{N \backslash S *}\right)\right]}{n !} \\
& =\frac{\sum_{S \in \Theta^{T}} \alpha_{S}[v(S)+(v(N)-v(S))]}{n !}=\frac{v(N) \sum_{S \in \Theta^{T}} \alpha_{S}}{n !} \\
& =v(N)
\end{aligned}
$$


(ii) The function defined by Equalities (1) and (2) satisfies Additivity*:

$$
\begin{aligned}
\phi_{i}(w+u, T) & =\frac{\sum_{S \in \Theta^{T}, i \in S} \alpha_{S} \varphi_{i}\left((w+u)^{S}\right)+\sum_{S \in \Theta^{T}, i \notin S} \alpha_{S} \varphi_{i}\left((w+u)^{N \backslash S *}\right)}{n !} \\
& =\frac{\sum_{S \in \Theta^{T}, i \in S} \alpha_{S} \varphi_{i}\left(w^{S}+u^{S}\right)+\sum_{S \in \Theta^{T}, i \notin S} \alpha_{S}\left[\varphi_{i}\left(w^{N \backslash S *}+u^{N \backslash S *}\right)\right]}{n !} \\
& \left.\left.=\frac{\sum_{S \in \Theta^{T}, i \notin S} \alpha_{S}\left[\varphi_{i}\left(w^{N \backslash S *}\right)+\varphi_{i}\left(u^{S}\right)\right]+}{n !} u^{N \backslash S *}\right)\right] \\
& =\phi_{i}(w, T)+\phi_{i}(u, T)
\end{aligned}
$$

(iii) As a direct consequence of Symmetry of the Shapley value, the function defined by Equalities (1) and (2) satisfies the Symmetry defined in Axiom 6.

(iiii) The function defined by Equalities (1) and (2) satisfies Commitment cost. Consider a unanimity game $v_{K}$. Since $K$ is a carrier of $v_{K}$ we can write using Equality (2):

$$
\phi_{T}\left(v_{K}, T\right)=\sum_{i \in T} \frac{\sum_{S \in \Theta^{T}, i \in S} \alpha_{S} \varphi_{i}\left(v_{K}^{S}\right)}{k !}
$$

with $\Theta^{T}=\{S \subset K: T \subset S\}$. Given the definition of $\varphi_{i}\left(v_{K}^{S}\right)$ we know that for each $S$ in $\Theta$ different from $K, \varphi_{i}\left(v_{K}^{S}\right)=0$. Otherwise $\varphi_{i}\left(v_{K}^{K}\right)=\varphi_{i}\left(v_{K}\right)=\frac{1}{k}$ and in that case $\alpha_{K}=(k-1) ! t$. Therefore:

$$
\phi_{T}\left(v_{K}, T\right)=\sum_{i \in T} \frac{(k-1) ! t \frac{1}{k}}{k !}=\frac{t^{2}}{k^{2}}
$$

Given that $\forall i \in K, \phi_{i}\left(v_{K}, K\right)=\varphi_{i}\left(v_{K}\right)=\frac{1}{k}, \phi_{T}\left(v_{K}, K\right)=\varphi_{T}\left(v_{K}\right)=\frac{t}{k}$. Therefore $\frac{t}{k} \phi_{T}\left(v_{K}, K\right)=\frac{t^{2}}{k^{2}}$ and Commitment Cost is verified.

Second step: I have now to prove that the function defined in Equalities (1) and (2) is the unique function which satisfies the four Axioms. Each game can be written as a linear combination of symmetric games $v_{K}$ (Shapley 1953 lemma 3). Therefore, by Additivity*, it is sufficient to show that the Axioms allow us to characterize the value for each symmetric game. Consider a symmetric game $v_{K}$ and a threshold $T$. By Efficiency*, since $K$ is a carrier of $v_{K}$, $\sum_{i \in K} \phi_{i}\left(v_{K}, T\right)=1$ or $\phi_{T}\left(v_{K}, T\right)+\phi_{N \backslash K}\left(v_{K}, T\right)=1$. By Symmetry*, deduce that $\forall i \in T, \phi_{i}\left(v_{K}, T\right)=\phi^{*}\left(v_{K}, T\right)$ and $\forall i \in K \backslash T, \phi_{i}\left(v_{K}, T\right)=\phi^{* *}\left(v_{K}, T\right)$ and hence:

$$
t \phi^{*}\left(v_{K}, T\right)+(k-t) \phi^{* *}\left(v_{K}, T\right)=1
$$

Therefore, by Commitment cost we know that $\phi_{T}\left(v_{K}, T\right)=\frac{t}{k} \phi_{T}\left(v_{K}, K\right)=$ $\frac{t}{k} \frac{1}{k}$ and $(k-t) \phi^{* *}\left(v_{K}, T\right)=1-t \frac{t}{k} \frac{c}{k}$. Therefore:

$$
\begin{aligned}
\phi_{i}\left(v_{K}, T\right) & =\frac{1}{k} \cdot \frac{t}{k}, \forall i \in K \\
\phi_{i}\left(v_{K}, T\right) & =\frac{1}{k}\left(1+\frac{t}{k}\right), \forall i \in N \backslash K
\end{aligned}
$$


In order to introduce some asymetries in the players' bargaining weights, Shapley (1953) proposed the family of weighted Shapley values. Each weighted Shapley value associates a positive weight with each player. These weights are the proportions in which the players share in symmetric games. In the case of the $T$-value, the weights are determined by the threshold, as described in the following corollary:

Corollary 1 For a given symmetric game $v_{K}$ and a threshold $T \subset K$, the T-value generates the weights:

$$
\omega_{i}=\frac{t}{k^{2}}, \forall i \in T \text { and } \omega_{i}=\frac{t+k}{k^{2}}, \forall i \in K \backslash T
$$

Note that, since $\frac{\omega_{j}}{\omega_{i}}=1+\frac{k}{t}$, for each pair $(i, j), i \in T, j \in K \backslash T$, the weight of $K \backslash T$-members increases with the size of $K$ relative to the size of $T$. Moreover, given that $k \geq t \geq 0$, when $t=0$ or when $t=k, \omega_{i}=\frac{1}{n}, \forall i \in N$ and we are back in the symmetric case of the Shapley value. Therefore, the non binding threshold as well as the strongest threshold do not affect the outcome of the negotiation.

Example 4 Consider the following symmetric game with three players $U=$ $\{1,2,3\}$,

$$
\begin{aligned}
v_{12}(S) & =1 \text { if } 12 \subset S \\
& =0 \text { otherwise }
\end{aligned}
$$

The Shapley value is: $\varphi_{1}\left(v_{12}\right)=\varphi_{2}\left(v_{12}\right)=\frac{1}{2} ; \varphi_{3}\left(v_{12}\right)=0$. When the threshold is $T=\{1\}$, the $T$-value is $\phi_{1}\left(v_{12},\{1\}\right)=\frac{1}{4} ; \phi_{2}\left(v_{12},\{1\}\right)=\frac{3}{4} ; \phi_{3}\left(v_{12},\{1\}\right)=0$

Example 5 Weighted majority games are characterized by the existence of "weights" $w_{i}$ such that it is never the case that $\sum_{i \in S} w_{i}>\sum_{N \backslash S} w_{i}$ and such that (see Shapley (1953) in Roth (1988) p. 39) for each carrier $N$ :

$$
\begin{gathered}
v(S)=n-s \text { if } \sum_{S} w_{i}>\sum_{N \backslash S} w_{i} \\
v(S)=-s \text { if } \sum_{S} w_{i}<\sum_{N \backslash S} w_{i}
\end{gathered}
$$

Let us compare $v(S)$ and $v(N)-v(N \backslash S)$. If $v(S)=n-s$ then $v(N \backslash S)=$ $-(n-s)$. Given that $v(N)=0$, we deduce that $v(S)=v(N)-v(N \backslash S)$. As a consequence, in weighted majority games, whatever the threshold is, the T-value coincides with the Shapley value.

Kalai and Samet (1987) proposed an axiomatic characterization of the family of weighted Shapley values introduced by Shapley (1953). They consider that the weights are associated with an ordered partition of $N$. Then, the family of 
weighted Shapley values is characterized by the way the ordered partition and the associated weights, in other words the weight system, generate a probabilistic distribution on the orders of $N$. One important characteristic of this probabilistic distribution is that it vanishes on each order which is not consistent with the ordered partition.

Hart and Kurz (1983) have proposed another value defined for a given coalition structure. But in this case the partition is not ordered and as a consequence, the CS-value proposed by Hart and Kurz does not satisfy Kalai and Samet's Axioms. However, in both cases the coalition structure limits the number of orders to appear. In the CS-value, the only orders to appear are orders consistent with the coalition structure: the orders in which the coalitions of the structure appear as blocks. But these blocks can appear in different orders.

In the case of the $T$-value, the threshold does not modify the probabilistic distribution on the orders of $N$, nor eliminate certain orders of $N$, but it superimposes different orders. To each order of $N$, corresponds a coalition which reaches the threshold and this determines two other set of orders: a set of orders of coalition $S$ and a set of orders of coalition $N \backslash S$. As a consequence, the $T$-value is an expected Shapley value.

\section{A more general definition of thresholds}

The definition of thresholds used in the previous section is rather restrictive. In this Section, I am going to propose a general definition of thresholds but, without giving an axiomatization for this general case. Instead, I will discuss the properties of the function.

Definition $1 A$ threshold is defined on an interval $I \subset \mathbb{R}_{+}$by a rule which associates to each threshold level $\tau \in I$, a set of coalitions $\Theta(\tau)$.

I will say that the coalitions in $\Theta(\tau)$ satisfy threshold $\tau$. Denote by $\underline{\Theta}(\tau)$ the subset of minimal coalitions in $\Theta(\tau): \underline{\Theta}(\tau)=\{S \in \Theta(\tau), \nexists K \in \Theta(\tau), K \subset S\}$. Note that coalitions in $\underline{\Theta}(\tau)$ are not necessarily disjoint. The threshold for which I can give an axiomatization of the $T$-value is the specific case in which there is a unique coalition in $\underline{\Theta}$.

Example 6 The rule which associates with each threshold level $\tau$ in the interval $[0 ; v(N)]$, a set of coalitions $\Theta(\tau)=\{S: v(S) \geq \tau\}$.

In what follows, for the simplicity of presentation, $\underline{\Theta}(\tau)$ will be called the threshold. For each given game $(N, v)$ and threshold $\underline{\Theta}(\tau)$, the $T$-value is denoted, for each player $i \in N$, by $\phi_{i}(v, \underline{\Theta}(\tau))$. Is it always the case that the threshold introduces a bias in the Shapley value? First, note that for a given rule, the threshold level is not necessary binding. In the previous example, if the threshold level is so low that each player alone can reach it, $\underline{\Theta}(\tau)=\{S: S \subset N\}$, or if it is so large that the grand coalition is the only coalition that can reach it, $\underline{\Theta}(\tau)=\{N\}$, then the $T$-value coincides with the Shapley value.

$$
\phi_{i}(v, N)=\varphi_{i}(v)
$$




$$
\phi_{i}(v,\{S: S \subset N\})=\varphi_{i}(v)
$$

When, whatever the threshold's level is, the $T$-value coincides with the Shapley value, I will say that the rule which defines the threshold is neutral .

Definition 2 A rule which defines a threshold on an interval $I$ is neutral if and only if $\forall \tau \in I, \phi_{i}(v, \underline{\Theta}(\tau))=\varphi_{i}(v), \forall i \in N$.

Now, let us generalize the $T$-value defined in Section 3 to thresholds as defined in Definition 1. The probabilistic description of thresholds is still available.

Definition 3 For a given game $v$ and a threshold $\underline{\Theta}(\tau)$, the $T$-value for each player $i \in N$ is given by:

$$
\phi_{i}(v, \underline{\Theta}(\tau))=\frac{\sum_{S \in \Theta(\tau), i \in S} \alpha_{S} \varphi_{i}\left(v^{S}\right)+\sum_{S \in \Theta(\tau), i \notin S} \alpha_{S} \varphi_{i}\left(v^{N \backslash S *}\right)}{n !}
$$

At this point, we can consider the properties of the $T$-value in this general case.

Proposition 2 The T-value for any general threshold satisfies Efficiency*, Additivity* and Symmetry*.

Proof. Same as before.

What can we say about the Commitment cost in this general context? The first Proposition of efficiency means that the players share the value of the grand coalition. But this also means that, if a given player's $T$-value is larger than her Shapley value, in other words if this player benefits from the introduction of threshold $\underline{\Theta}(\tau)$, this occurs at the expense of another player who loses. The following Proposition, which is a direct consequence of the decomposition of the Shapley value, shows that this may not always be the case. Indeed, if the rule that defines the threshold is such that all the coalitions of a given size $\widetilde{m}$ reach the threshold, then, the $T$-value coincides with the Shapley value.

Proposition 3 Each threshold rule such that $\exists \widetilde{m}<n$ and $\underline{\Theta}(\tau)=C_{\widetilde{m}}$, is neutral.

Proof. This is straightforward. If $\exists \widetilde{m}<n$ such that $\underline{\Theta}(\tau)=C_{\widetilde{m}}$, each order is associated with a coalition of size $\widetilde{m}$ and each coalition $M$ of size $\widetilde{m}$ appears in $\alpha_{M}=(n-\widetilde{m}) ! \tilde{m}$ ! orders. Therefore, using the decomposition of the Shapley value we check that:

$$
\phi_{i}\left(v, C_{\tilde{m}}\right)=\frac{(n-\widetilde{m}) ! \widetilde{m} !\left[\sum_{S \in C_{\tilde{m}}^{i}} \varphi_{i}\left(v^{S}\right)+\sum_{S \in C_{\tilde{m}}^{-i}} \varphi_{i}\left(v^{N \backslash S *}\right)\right]}{n !}=\varphi_{i}(v) .
$$


An obvious example of threshold rule which does not allow any manipulation of the Shapley value is a rule defined as a number of players. Indeed, in that case, the previous Proposition applies and the $T$-value cannot be different from the Shapley value.

Corollary 2 The threshold rule which associates to each threshold level $\tau$ in the interval $[1 ; n]$, a set of coalitions $\underline{\Theta}(\tau)=C_{\geq \tau}$ is neutral.

Proof. This is a direct consequence of the previous Proposition.

\section{Conclusion}

In this paper, the $T$-value is proposed to represent the prospective outcome or "prospect" of a negotiation when the players' value can only be realized if a threshold is reached. The threshold is associated with a minimal coalition $T$ and it is reached by every coalition that contains $T$. The analysis conducted in this paper allows one to deal with many real situations. Consider for example the case of international negotiations of treaties mentioned in the introduction. The minimum participation constraint, which is obviously a threshold necessary for the treaty to be implemented, can be defined in three different ways: as a set of countries, a number of countries or a level of the global target. In this paper, the cases I consider in order to define the $T$-value corresponds to the first definition of thresholds. Then, the axiomatization reflect the idea that the players who are needed for the threshold to be reached have to pay a cost for their higher commitment in the negotiation. In an attempt to extend the definition of the $T$-value, I proved that when the threshold is a number of countries, the $T$-value coincides with the Shapley value. The extention of the $T$-value to a general definition of threshold will be the object of further research. 


\section{Appendix}

\section{Proof of Proposition 1:}

First, I need to introduce some notation and to make two remarks. Denote by $C_{m}^{i}, C_{<m}^{i}, C_{>m}^{i}$ the sets of coalitions to which player $i$ belongs and of size, respectively, equal to, smaller than or equal to and larger than $m$. Therefore:

$$
C_{\leq m}^{i}=\cup_{k=1}^{m} C_{k}^{i} \text { and } C_{>m}^{i}=\cup_{k=m+1}^{n} C_{k}^{i}
$$

On the other hand $C_{m}^{-i}, C_{<m}^{-i}, C_{>m}^{-i}$ are the sets of coalitions to which player $i$ does not belong and of size, respectively, equal to, smaller than or equal to or larger than $m$. Denote by $E_{M}^{i}$ the set of coalitions included in coalition $M$, to which player $i$ belongs: $E_{M}^{i}=\{S: i \in S \subset M\}$. Therefore, $\cup_{M \in C_{m}^{i}} E_{M}^{i}$ is the set of all coalitions to which player $i$ belongs and which are included in coalitions of size $m$. Comparing this set $\cup_{M \in C_{m}^{i}} E_{M}^{i}$ with the set $C_{\leq m}^{i}$ we can make the following remark:

Remark 1 Each coalition $S \in C_{\leq m}^{i}$ appears $\complement_{n-s}^{m-s}=\frac{(n-s) !}{(m-s) !(n-m) !}$ times in $\cup_{M \in C_{m}^{i}} E_{M}^{i}$.

Example 7 Consider the case in which $N=\{1,2,3\}, m=2$ and $i=1$. Then, $C_{\leq 2}^{1}=\{1,12,13\}$ and $\cup_{M \in C_{m}} \cup_{M \in C_{m}^{1}} E_{M}^{1}=\{1,12,1,13\}$. The singleton $\{1\}$ appears once in $C_{\leq 2}^{1}$ and twice in $\cup_{M \in C_{m}^{1}} E_{M}^{1}$.

Denote by $E_{M}^{i *}$ the set of coalitions $S$ to which player $i$ belongs and in which $M$ is included: $E_{M}^{i *}=\{S: M \subset S, i \in S\}$. Now, comparing $C_{>m}^{i}$ and $\cup_{M \in C_{m}^{-i}} E_{M}^{i *}$ we can make the second following remark:

Remark 2 Each coalition $S \in C_{>m}^{i}$ appears $\complement_{s-1}^{m}=\frac{(s-1) !}{m !(s-m-1) !}$ times in $\cup_{M \in C_{m}^{-i}} E_{M}^{i *}$.

Example 8 Consider the case in which $N=\{1,2,3,4\}, m=2$. Then, $C_{>2}^{1}=$ $\{123,124,134,1234\}$ and $\cup_{M \in C_{m}^{-1}} E_{M}^{1 *}=E_{23}^{1 *} \cup E_{24}^{1 *} \cup E_{34}^{1 *}$ with $E_{23}^{1 *}=\{123,1234\}$, $E_{24}^{1 *}=\{124,1234\}$, and $E_{34}^{1 *}=\{134,1234\}$.

For each size of coalition $m$, we can write:

$$
\begin{aligned}
n ! \varphi_{i}(v)= & \sum_{S \in C_{\leq m}^{i}}(s-1) !(n-s) !(v(S)-v(S \backslash i)) \\
& +\sum_{S \in C_{>m}^{i}}(s-1) !(n-s) !(v(S)-v(S \backslash i))
\end{aligned}
$$


I am going to use the first previous remark in what follows:

$$
\begin{aligned}
& \sum_{M \in C_{m}^{i}} m ! \varphi_{i}\left(v^{M}\right) \\
= & \sum_{M \in C_{m}^{i}} \sum_{S \subset M}(s-1) !(m-s) !(v(S)-v(S \backslash i)) \\
= & \sum_{S \in \cup E_{M}^{i}}(s-1) !(m-s) !(v(S)-v(S \backslash i)) \\
= & \sum_{S \in C_{\leq m}^{i}}(s-1) !(m-s) !\left[\frac{(n-s) !}{(m-s) !(n-m) !}\right](v(S)-v(S \backslash i))
\end{aligned}
$$

And therefore:

$$
\begin{aligned}
& \sum_{M \in C_{m}^{i}} \varphi_{i}\left(v^{M}\right) \\
= & \frac{1}{m !(n-m) !} \sum_{S \in C_{\leq m}^{i}}(s-1) !(n-s) !(v(S)-v(S \backslash i))
\end{aligned}
$$

The second remark will be used in what follows. First note that $\varphi_{i}\left(v^{N \backslash M *}\right)$, which is for each player $i \in N \backslash M$ :

$$
\varphi_{i}\left(v^{N \backslash M *}\right)=\frac{\sum_{S \subset N \backslash M, i \in S}(s-1) !(n-m-s) !(v(S \cup M)-v(S \backslash i \cup M))}{(n-m) !}
$$

can be written:

$$
\varphi_{i}\left(v^{N \backslash M *}\right)=\frac{\sum_{S \in E_{M}^{i *}}(s-m-1) !(n-s) !(v(S)-v(S \backslash i))}{(n-m) !}
$$

Therefore, using the remark:

$$
\begin{aligned}
& (n-m) ! \sum_{M \in C_{m}^{-i}} \varphi_{i}\left(v^{N \backslash M *}\right) \\
= & \sum_{M \in C_{m}^{-i}} \sum_{S \in E_{M}^{i *}}(s-m-1) !(n-s) !(v(S)-v(S \backslash i)) \\
= & \sum_{S \in C_{>m}^{i}}(s-m-1) !(n-s) !\left[\frac{(s-1) !}{m !(s-m-1) !}\right](v(S)-v(S \backslash i))
\end{aligned}
$$

and:

$$
\begin{aligned}
& \sum_{M \in C_{m}^{-i}} \varphi_{i}\left(v^{N \backslash M *}\right) \\
= & \frac{1}{m !(n-m) !} \sum_{S \in C_{>m}^{i}}(n-s) !(s-1) !(v(S)-v(S \backslash i))
\end{aligned}
$$


Finally, adding (4) and (5) gives:

$$
\begin{aligned}
& m !(n-m) !\left[\sum_{M \in C_{m}^{i}} \varphi_{i}\left(v^{M}\right)+\sum_{M \in C_{m}^{-i}} \varphi_{i}\left(v^{N \backslash M *}\right)\right] \\
= & \sum_{S \in C_{\leq m}^{i}}(s-1) !(n-s) !(v(S)-v(S \backslash i)) \\
& +\sum_{S \in C_{>m}^{i}}(n-s) !(s-1) !(v(S)-v(S \backslash i)) \\
= & n ! \varphi_{i}(v)
\end{aligned}
$$




\section{References}

Bagnoli M. and B. Lipman (1989), "Provision of Public Goods: Fully Implementing the Core through Private Contributions", Review of Economic Studies, 56, 583-601.

Barrett S., Environment \& Statecraft, The Strategy of Environmental Treatymaking, Oxford University Press, 2003.

Maskin E. (2003), "Bargaining, coalitions and externalities".

Hart and Kurz, (1983), "Endogenous Formation of Coalitions", Econometrica, Vol. 51, No 4, 1047-1064.

Ledyard J. (1995), "Public Goods: A survey of Experimental Research", in Kagel, J. and Roth, A. (eds.), The Handbook of Experimental Economics, Princeton University Press.

Littlechild, S. C., and G. F. Thompson, (1977), "Aircraft Landing Fees: A Game Theory Approach", Bell Journal of Economics, Vol. 8, n 1, 186-204.

Palfrey T. and Rosenthal H. (1984), "Participation and the Provision of Discrete Public Goods", Journal of Public Economics, 24, 171-193.

Perez-Castrillo D., D. Wettstein (2001) "Bidding for the Surplus: A Noncooperative Approach to the Shapley Value", Journal of Economic Theory, 100, 274-294.

Roth A. E. (1988), The Shapley value: Essays in honnor of Lloyd S. Shapley, Cambridge University Press, Cambridge.

Shapley, (1953), "A Value for n-Person Games", in Contributions to the Theory of Games, Vol. II, ed. By Kuhn and Tucker. Priceton; Princeton University Press, 307-317.

Von Neumann and Morgenstern (1944), Theory of Games and Economic Behavior, Princeton, Princeton University Press. 